\title{
LA EDICIÓN DE PHILIPPO DE GIUNTA DEL BELLUM GALLICUM DE CÉSAR (FLORENCIA 1514) ${ }^{1}$
}

\author{
ANTONio M ORENO HERNÁNDEZ \\ UNED. Madrid \\ anmoreno@flog.uned.es
}

\section{RESUMEN}

En 1514, al año siguiente de que viera la luz en el taller de P. Manuzio la edición latina de los Commentarii de César preparada por Giovanni Giocondo, Philippo de Giunta publicó en Florencia una nueva edición de la obra cesariana. El propósito de este artículo es analizar la relación entre ambas ediciones desde el punto de vista crítico sobre el texto del Bellum Gallicum. La edición de Giunta no sólo emula los rasgos compositivos característicos de la edición aldina. sino que adopta como base el texto establecido por Giocondo, si bien, bajo la apariencia mimética de la edición giuntina, se revela la existencia de una incipiente labor crítica consistente en la revisión de un número considerable de variantes del texto aldino a la luz de las lecturas de la tradición manuscrita y editorial anterior. poniendo de manifiesto un claro ejemplo de emendatio ope codicum ef editionum.

Palabras clave: Julio César: Bellum Gallicum; Philippo de Giunta: tradición postincunable.

\section{ABTRACT}

In 1514, one year after Aldo Manuzio published the latin edition of the Caesars Commentarii established by Giovanni Giocondo. Philippo di Giunta brought to light in Florence another edition of these books. The aim of this article is to analyse the rela- 
tionship between these two postincunable editions from a critical point of view on the text of Bellum Gallicum. Giunta's edition not only seems to emulate the compositive features of the Aldine edition, but the former assumes as basis the text of the the latter. However, below this mimetic appearance, it is possible to prove the existence of an incipient critical task in the florentine edition in order to revise a considerable number of the variants of the Aldine edition, in the light of lectiones that come from the manuscript and editorial previous tradition, revealing an example of the emendatio ope codicum et editionum.

Key words: Iulius Caesar; Bellum Gallicum; Philippo di Giunta; postincunable tradition.

\section{INTRODUCCIÓN}

A comienzos del siglo XVI el interés por los Commentarii de César cobra un nuevo impulso gracias a la actividad de una nueva generación de impresores y humanistas italianos que acometen varias empresas de edición de las obras cesarianas. Sólo en los primeros años del Quinientos se suceden las ediciones de Philippo Beroaldo (Bolonia 1504) ${ }^{2}$, de Philippo de Giunta (Florencia 1508) ${ }^{3}$, y de Agustino de Zannis de Portesio (Venecia 1511) 4 .

Pero es en 1513 cuando ve la luz una edición de gran trascendencia filológica para la historia del texto cesariano: la edición preparada por Giovanni Giocondo para Aldo Manuzio (a), a partir de la cual se conforma una nueva vulgata de los Commentarii en las primeras décadas del s. XVI. Pues bien, a los pocos meses de su publicación, en agosto de 1514, se publica en Florencia una nueva edición de la obra cesariana en el taller de Philippo de Giunta ${ }^{5}$, uno de los impresores más destacados de la época, cuya labor editorial ${ }^{6}$

1 Este artículo se inscribe dentro del Proyecto de Investigación «Estudio filológico de la tradición textual y literaria de la obra de Julio César en España (II)», financiado por la DGl (Ref. HUM2005-00442).

${ }^{2}$ Commentarii Caesaris, recogniti per Philippum Beroaldum... Impressit Bononiae Benedictus Hectoris, anno salutis 1504. Cal. aprilis... Bononiae, 1504 (en fol.); posteriormente reimpresa por Balthazar de Gabiano en Lión 1508 (Commentarii Caesaris, recogniti per Philippum Beroaldum. Lugduni, Balthazar, 1508, en 8..$^{\circ}$ y 1512 (Commentarii Caesaris, recogniti per Philippum Beroaldum, Lugduni, Balthazar, 1512, en 8..$^{\circ}$.

${ }^{3}$ A cargo de Luca della Robbia (Commentaria Caesaris, edente Luca Robia. Impressum Florentiae, opera... Philippi Giuntae,... anno... D. octavo supra mille, mense aprili).

${ }^{4}$ Caii Julii Caesaris commentaria seculorum injuria antea difficilia \& valde mendosa. Venetiis per Augustinum de Zannis de Portesio. Anno reconciliatae nativitatis M.D.XI die XVII. Augusti. Reeditada en 1517 (Caii Julii Caesaris Commentaria... nunc primum a viro docto (Luca Olchinensi) expolita et optime recognita. Venetiis, per Augustinum de Zannis de Portesio, anno... 1517, die XIII. Junii. Edición de referencia: Toledo, Biblioteca de Castilla La Mancha, Fondo Antiguo, Res. 308).

${ }^{5}$ Commentaria Caesaris, prius a Jocundo impressioni data, posterius a nobis diligentissime revisa.... Ex officina P. de Giunta (edición de referencia: París, BNF J-13272).

- Sobre la obra de Filippo en el ámbito del taller familiar de los Giunta, sigue siendo de referencia la «Notice sur la famille des Junte et liste sommaire de leurs éditions jusqu'en 1550" que apareció como apéndice a A. Renouard, Annali delle edizioni aldina, Bolonia 1953 (facs. della 3, Paris, 1834, cf. New Castle 1991), pp. VILXVIII, así como las ediciones y estudios de los anales de su taller, cf. D. Decia, I Giunti tipografi editori di Firenze, 1497-1570 (Parte I: Annali 1497-1570, con un saggio introduttivo di Renato Delfiol; Parte II: Commentario agli Annali 1497-1570), Florencia 1978; P. Camerini, Annali dei Giunti: Venezia, Florencia, 1962-1963, 
se extiende desde 1497 a 1517 y comprende más de un centenar de ediciones ${ }^{7}$, entre las cuales priman textos latinos, griegos e italianos de corte humanístico, así como algunos textos legales y religiosos.

La edición de Giunta de 1514 -que denominaremos g-, cuyo valor crítico se puso de manifiesto a raíz de su inclusión en la célebre Tabula coniecturarum de Meusel $^{8}$ y también por la consideración de algunas de sus correcciones por parte de la edición oxoniense de Du Pontet ${ }^{9}$, no sólo emula los rasgos editoriales característicos de la imprenta aldina - la tipografía cursiva y el formato en $8 .^{\circ}-$, sino que adopta como base el texto establecido por Giocondo, como reza en la misma portada de la edición:

Commentaria Caesaris, prius a Jocundo impressioni data, posterius a nobis diligentissime reuisa et cum exemplaribus eiusdem collata, restitutis omnibus locis, quae per incuriam ab iis qui ea impresere uel relicta uel errata fuerunt...

El propósito de este artículo es determinar, sobre el texto del Bellum Gallicum, la relación entre esta edición giuntina y la edición del taller aldino de 1513 y, concretamente, en qué consisten las alteraciones de aquélla respecto a ésta, pues el florentino parece dejar patente en este comentario del título su voluntad de no reproducir el texto de la edición preparada por el ingeniero y humanista Giocondo ${ }^{10}$ sino revisarlo cuidadosamente

2 v.; L.S. Camerini, I Giunti tipografi editori di Firenze, 1571-1625: Annali inediti con un appendice sui bibliografi dei Giunti, Florencia 1979; A.M. De Florentina Iuntarum typographia eiusque censoribus ex qua Graeci, Latini, Tusci scriptores ope codicum manuscriptorum a viris clarissimis pristinae integritati restituti in lucem prodierunt, Lucca 1791 (Ridgewood 1965); W.A. Pettas, The Giunti of Florence: merchant publishers of the sixteenth century (With a checklist of all books and documents publ. by the Giunti in Florence from 1497 to 1570, and with the texts of 29 documents, from 1427 to the 18th century, San Francisco), Rosenthal, 1980; F.J. Norton, Italian Printers 1501-1520, Londres 1958, pp. 29-31; cf. también los trabajos de F. Ascarelli, M. Menato, La Tipografia del' 500 in Italia, Florencia 1989, 271-273; P.C. Marani, «I Giunti e il Rinascimento fiorentino», L'Esopo 1, 1979, pp. 51-55; C. Di Filippo Bareggi, «Giunta, Doni, Torrentino: tre tipografie fiorentine fra Reppublica e Principato», Nuova Rivista Storica 58, 1974, pp. 318-348.

7102 según el catálogo incompleto de Renouard, y en torno a 40 de sus herederos, hasta 1520 .

${ }^{8} \mathrm{H}$. Meusel, recoge algunas de las correcciones de la edición de $1514-$ y no la de edición giuntina de 1508- en el Apéndice de su Lexicon Caesarianum (Tabula Coniecturarum), Berlín 1887-1893, a pesar de que, según confiesa, él mismo no ha realizado directamente la colación (Ipse hanc edit. non uidi, cf. op. cit., p. VI).

${ }^{9}$ R. du Pontet, C. I. Caesaris Commentariorum, I, De bello Gallico, Oxford 1900 (múltiples reimpr. posteriores). Du Pontet distingue en su Conspectus entre la edición giuntina de 1508 y la de 1514 , si bien algunas de las atribuciones que aparecen en su aparato crítico no son correctas: así 3.23.3 du Pontet atribuye la corrección diduci -asumida unánimemente por todos los editores modernos-a Giunta 1514, siguiendo probablemente la Tabula coniecturarum de Meusel (quien utiliza, según su conspectus, la edición Juntina de 1514), frente a deduci de la tradición manuscrita. Sin embargo, la corrección se encuentra ya en la edición de Giunta de 1508, como recogen los aparatos críticos de Klotz, Seel y Hering. La corrección se encuentra así mismo en la edición de Beroaldo de 1512 y está igualmente incorporada en la ediciones aldinas de 1513 -modelo de g- y en la de 1519 , y patir de ellas, se encuentra generalizada en las ediciones postincunables.

${ }^{10}$ Giocondo había preparado la edición de César tras su estancia en París, donde tuvo acceso a nuevos manuscritos de los Commentarii, llevando a cabo una minuciosa labor de emendatio de manuscritos -en general recentiores - y ediciones anteriores. Sobre los avatares de su trayectoria, cf. L. A. Ciapponi «Appunti per una biografia di Giovanni Giocondo da Verona», Italia Medioevale e Umanistica 11, 1961, 131-158, y R. Brenzoni, Fra Giovanni Giocondo Veronese: Verona 1435 - Roma 1515, Florencia, 1960, pp. 129-149. Su labor filológica ha sido estudiada en particular sobre su edición de Vitrubio (L. A. Ciapponi, «Fra Giocondo da Verona and His Edition of Vitruvius", The Journal of the Warburg and Courtauld Institutes, 47, 1984, pp. 72-90, y «Il "De Architectura" di Vitruvio nel primo umanesimo", Italia medioevale e umanistica 3, 1969, pp. 59-99, V. Jurẽn, "Fra 
(diligentissime revisa) ${ }^{11}$ para restituir todos aquellos pasajes deficientemente editados por el impresor anterior - en clara alusión a Aldo, sin mencionarlo ${ }^{12}$ - ¿ ¿Se trata de una simple declaración de intenciones? ¿Responde este comentario de Philippo de Giunta a una voluntad real de replantearse el texto de la edición aldina, o es un mero recurso para evitar la acusación de imitación o plagio? No hay que olvidar que este impresor florentino fue uno de los grandes imitadores y rivales de Aldo Manuzio ${ }^{13}$, quien procuró buscar el respaldo de la autoridad papal para evitar el plagio de sus tipos y edicione ${ }^{14}$, en el marco de la extraordinaria eclosión editorial que viven en esta época ciudades como Venecia, Florencia y Roma ${ }^{15}$.

Bien es cierto que el interés de Philippo de Giunta por César se había materializado 6 años antes, en una primera edición de los Commentarii preparada por Lucca della Robbia $^{16}$, uno de los colaboradores habituales del taller florentino ${ }^{17}$, pero resulta muy reve-

Giovanni Giocondo et le début des études vitsuviennes en France", Rinascimento ( $2^{\text {nd }}$ ser.) 14, 1974, pp. 102-116, P.N. Pagliara, «Una fonte di illustrazioni del Vitruvio di Fra' Giocondo», Ricerche di storia dell' arte 6, 1977, pp. 113-20; y F. Granger, «Fra Giocondo's Manuscripts of Vitrubius», The Times Literary Supplement 28, 1929, p. 241), así como su interés por la epigrafía (L.A. Ciapponi, «A Fragmentary Treatise on Epigraphic Alphabets by Fra Giocondo da Verona», Renaissance Quarterly 32, 1979, pp. 18-40, y M. Koortbojian, «Fra Giovanni Giocondo and his epigraphic methods», Kolner Jarhbuch 26, 1993, pp. 49-55) y por las matemáticas (L. A. Ciapponi, «Disegni ed appunti di matematica in un codice di fra Giocondo da Verona (Laur. 29,43)», Vestigia. Studi in onore di Giuseppe Billanovich, Bd. 1, Roma, 1984; A. Tura, "Codici di matematica di Fra Giocondo", Bibliothèque d' Humanisme et Renaissance 61, 1999, pp. 701-711.

$"$ Este vocabulario forma parte del léxico habitual humanístico para referirse a la intervención en el texto mediante correcciones de diversa índole. Cf. S. Rizzo, II lessico filologico degli Umanisti, Roma 1973, pp. 243300. Sobre la naturaleza y alcance de estas prácticas conjeturales durante las primeras etapas de la imprenta, sigue siendo fundamental E.J. Kenney, The Classical Text. Aspects of Editing in the Age of the Printed Book, Berkeley - Londres 1974.

${ }_{12}$ El pasaje revela, a nuestro entender, una crítica implícita al trabajo de Aldo en el competitivo mundo de las imprentas humanísticas de comienzos del s. XVı. A los pocos meses de publicarse la edición aldina -recordemos que el prefacio a los estudiosos de Aldo está fechado en diciembre de 1513 - se publica esta edición florentina de 1514 .

${ }^{13}$ Cf. F. J. Norton, Italian Printers..., op. cit., p. 30.

${ }^{14}$ De hecho la edición aldina de 1513 reproduce, tras los prefacios iniciales, el exemplum del Papa Alejandro VI, concediendo a Aldo la licencia exclusiva sobre sus tipos y ediciones, así como las penas y el castigo de excomunión para los autores de plagios.

15 Sobre el ambiente intelectual en el que emerge la labor de Aldo y sus relaciones con otros talleres italianos de la êpoca, cf. M. Lowry, The World of Aldus Manutius: Business and Scholarship in Renaissance Venice, Nueva York (Il mondo di Aldo Manuzio: affari e cultura nella Venezia del Rinascimento. vers. it., Roma 1984, 2000); M. Davies, Londres 1995; C. Dionisotti, Milán 1995; D. S. Zeidberg (ed.), Aldus Manutius and Renaissance Culture. Essays in Memory of Franklin D. Murphy. Florencia 1998; O. Orlandi, Aldo Manuzio editore, Milán 1975; P. F. Grendler, Aldus Manutius: Humanist, teacher, and printer, Providence, R. I. 1984; P. Scapecchi, Aldo Manuzio, suoi libri, i suoi amici tra XV e XVI secolo: libri, biblioteche e guerre in Casentino, Florencia 1994; J. Crichton, Aldus Manutius, the printer of the Renaissance, Los Angeles 1938; M. Dazzi, Aldo Manuzio e il dialogo veneziano di Erasmo, Vicenza 1969.

${ }^{16}$ Commentaria Caesaris. (Auli Hirtii, vel Oppii, libri de hello Alexandrino, de hello Africo, de bello Hispaniensi. Edente Luca Robia). In fine: Impressum Florentiae, opera... Philippi Giuntae,... anno... D. octavo supra mille, mense aprili.

${ }^{17}$ Su colaboración con Giunta, en una tarea que conjuga la labor de corrector y editor, dio lugar a la publicación de varios obras de clásicos latinos, además del César de 1508: Quinto Curcio (1507, en 8\%), Tusculanae Quaestiones (1508) y De officiis (1508 y 1513), asi como los Libri Oratorii (1514), se produjo tras el fallecimiento de Benedetto Riccardini, más conocido como «Benedictus Philologus», quien trabajó para Giunta desde 1497 hasta su muente en 1506. Sobre los colaboradores de Philippo. Cf. el Apéndice de A. A. Renouard, «Notice sur la famille des Junte»... op. cit., pp. XI-XI. 
lador que el impresor florentino lleve a cabo una segunda, que no toma como base el texto de la de su propio taller de 1508 - que, sin embargo, ya incorporaba el formato en $8 .^{\circ}$ y la tipografía cursiva, imitación de la aldina-, sino que se presenta abiertamente como deudora de la edición de Giocondo.

\section{La VinCUlaCión CON LA EDICIÓN DE Giocondo de 1513 (a)}

$\mathrm{Si}$ atendemos a los elementos paratextuales que acompañan al texto de los Commentarii, podemos advertir que Philippo de Giunta ha eliminado de su edición cualquier alusión explícita a Aldo y a los prefacios con que éste iniciaba su edición cesariana de $1513^{18}$; tampoco reproduce el prefacio de Giocondo, en el cual da cuenta de los avatares de su edición ${ }^{19}$, pero sí incorpora otros dos elementos de la edición veneciana: en primer lugar, las ilustraciones - con diversas modificaciones en el trazado de las xilografíasacompañadas del comentario correspondiente de diversos pasajes preparados por el ingeniero veronés que recogía igualmente a la edición aldina ${ }^{20}$, añadiendo una ilustración más, con la descripción de Hispania; y, en segundo lugar, el índice de nombres en latín y francés de la edición aldina.

El formato de ambas ediciones guarda, por otro lado, estrechas afinidades que no sólo se aprecian en la tipografía cursiva y en el formato en $8 .^{\circ}$ - elementos que ya había utilizado Giunta en la edición cesariana de 1508 , que denominaremos $\mathbf{i}-$, sino también en la presentación del texto latino de forma exenta, sin las acotaciones marginales marginales con que éste se acompañaba habitualmente en la ediciones incunables italianas - desde la de Treviso 1480 - y en su disposición contínua, rasgo propio de la tradición manuscrita e incunable —que provocaba el denominado «efecto mancha» en las primeras ediciones-, pues la edición aldina todavía desconoce la organización del texto en parágrafos.

Como es sabido, el proceso de fijación de la tipografía propicia en la imprenta aldina una codificación bastante regular de todos los aspectos relativos a la presentación impresa del texto: así la obra refleja unos criterios de composición tipográfica muy homogéneos, tanto en el formato de línea como en el de caja, con una media de 45 tipos fundidos por línea y treinta líneas por página. Así mismo la edición refleja el esfuerzo de los impresos aldinos por el establecimiento de unas grafías estables tanto para la notación de las palabras latinas y la regularidación de los nombres propios como en el uso de la mayúscula, que tiende a restringirse a la inicial de éstos últimos y al comienzo de oración tras punto. Así mismo se aprecia el esfuerzo por establecer una puntuación que distingue con claridad el uso del punto y de la coma, que son los dos únicos signos utilizados con regularidad.

Así mismo Giunta maneja unos criterios de composición tipográfica muy similares a los aldinos, tanto en el formato de línea como en el de caja: la edición de 1513 viene a pre-

${ }_{18}$ El primer prefacio está dirigido al lector (Aldus lectori S.) y el segundo a los estudiosos (Aldus studiosis $S$.).

${ }^{19}$ Prefacio de Giovanni Giocondo, dedicado a Giuliano de Medici (Ioan. Iucundus Veronen. Iuliano Medi(i S.P.D.).

20 5 grabados originales de Giocondo y sus correspondientes comentarios: la construcción del puente sobre el Rin (4.17); la fortificación de Avarico, capital de los bitúriges (7.22-23); la fortificación que preparó César para el cerco de Alesia (7.62-73); la forticación de Uxeloduno (8.40); y la descripción de Massilia (B.C. 2.9). 
sentar una media de 45 tipos fundidos por línea y treinta líneas por página, cifras que se mantienen habitualmente en la de 1514, de manera que en ocasiones se advierte la coincidencia de final de línea e incluso de página ${ }^{21}$, ni son extrañas secuencias coincidentes de finales de línea ${ }^{22}$, si bien es más habitual que $\mathrm{g}$ procure evitar la coincidencia completa, por lo que la situación más frecuente es la de la discoincidencia entre ambas ediciones ${ }^{23}$.

Desde el punto de vista textual, el cotejo entre la edición de Giunta 1514, que denominaremos g y la edición aldina de 1513 (a) permite comprobar la profunda dependencia de aquélla respeto a ésta, una dependencia que se refleja en la extrema fidelidad con que g sigue el texto preparado por Giocondo, cuyos principales rasgos son éstos:

a) La sedimentación de muchos elementos de la tradición manuscrita e impresa anterior.

b) La difuminación del perfil textual característico de las dos clases ( $\alpha$ y $\beta$ ) que conforman la historia del texto a la luz de los testimonios de mayor valor crítico para la constitutio textus.

c) La intervención constante en la tradición manuscrita para incorporar correcciones que, de acuerdo con el Prefacio de Giocondo, restituyan el esplendor (nitorem) del texto cesariano.

En efecto, si analizamos el texto desde el punto de vista de sus relaciones con las dos grandes clases ( $\alpha$ y $\beta$ ), podemos comprobar que la edición de Giunta secunda escrupulosamente a la aldina.

En cuanto a la denominada clase $\alpha$, la edición giuntina sigue a la aldina, que se muestra irregular en el tratamiento de sus rasgos textuales distintivos: de las cinco omisiones, a no comparte tres de ellas, y sólo sigue a $\alpha$ en los otros dos casos:

a) a no se ve afectado por las siguientes omisiones que caracterizan a la clase $\alpha$ :

\subsection{2 et ex propinquitate pugnare edd.pr. a $\mathbf{g}$ om. $\alpha$}

7.12.2 Biturigum positum in uia Nouiodonum edd. pr. a g om. $\alpha$

8.11.1 nisi a maiore - 12.2 circumuentos adgrederetur edd.pr. a g om. $\alpha$

b) a comparte dos omisiones que caracterizan a la clase $\alpha$ :

\subsection{2 et si quos intercipere potuerunt om. $\alpha$ edd.pr. a $\mathbf{g}$}

7.45.2 mulorumque produci deque his stramenta om. $\alpha$ edd. pr. a $\mathbf{g}$

Si ahora hacemos la misma comprobación con las lecturas distintivas de la clase $\beta$, advertimos que la edición aldina tampoco comparte las dos omisiones más significativas de esta clase, rasgo que adopta igualmente la edición de Giunta:

21 Cf. 7.77 .3 authoritatis // Nihil constituye el final de fol. $99 \mathrm{v}$ en a y del fol. $96 \mathrm{r}$ en $\mathrm{g}$.

22 Cf. fol. $100 \mathrm{r}$ de a y fol. $96 \mathrm{v}$ de $\mathrm{g}$.

23. Este procedimiento, la coincidencia en renglón y plana, es un recurso habitual en Jas primeras ediciones para reproducir con mayor fidelidad a su modelo, como ocurre en la edición de los Commentarii preparada e el taller de Juan de Burgos en 1491, que sigue a la edición veneciana de Octavianus Scotus de 1482, cf. A. Moreno Hernández, «La edición incunable del Bellum Gallicum de Julio César (Burgos 1491)», Cuadernos de Filología Clásica (Estudios Latinos) 22, 2002, pp. 9-42; y «Identificación de dos estados en la edición de Julio César del Bellum Gallicum (Burgos 1491)», Epos 17, 2001, pp. 13-27. 
3.9.10 auxilia....arcessunt $\alpha$ edd.pr. a g om. $\beta$

7.77.13 cuius rei...iudicarem $\alpha$ edd. pr. a g om. $\beta$

Por consiguiente, $\mathrm{g}$ reproduce el comportamiento textual de a, respetando muchas de las conjeturas propuestas por primera vez en esta edición, como evidencia este ejemplo:

\section{1 .5 oceanum $\omega$ edd. pr. : oceano a g edd. post.}

Para justificar la lectio recepta de oceanum, las ediciones incunables se ven obligadas a reinterpretar la puntuación del pasaje, añadiendo un punto ante occeanum para hacerlo depender de attingit y haciendo desaparecer entre oceanum y finibus: Garumna flumine. Oceanum finibus Belgarum attingit). La corrección de a viene a restablecer la sintaxis de la secuencia de ablativos Garumna flumine, oceano, finibus Belgarum dependiente de continetur, y a la vez revisa la puntuación de las ed. pr:: continetur Garumna flumine, oceano, finibus Belgarum; attingit....). La propuesta de a es adoptada inmediatamente por $\mathbf{g}$ y por las ediciones posteriores $\left(\mathbf{a}^{2}\right.$ y $\left.\mathbf{a}^{3}\right)$ y es asumida por todos los editores modernos, así como su propuesta de corrección de la puntuación.

Así mismo, La afinidad de $\mathbf{g}$ con a llega al extremo de reproducir las tendencias gráficas de la edición aldina (6.3.3 negocio a g), el uso de las mayúsculas (incluso en usos inapropiados (cf. 6.2.3 de Bello a g), el corte de palabras (7.52.1 sibiipsi a g) y la puntuación (8.25.1 ciuitatem, Cum in omnes a g).

\section{ADOPCIÓN DE LAS CORRECCIONES A LA EDICIÓN ALDINA $\left(\mathbf{a}^{c}\right)$}

Pero la edición de 1514 no se limita a reproducir, en buena medida, el texto de Giocondo, sino que introduce un buen número de las correcciones que fueron añadida en un pliego sin numerar a la edición de 1513 bajo la denominación de errata $^{24}$ y que denominaremos $\mathbf{a}^{\mathfrak{c}}$, y que se elaboraron probablemente durante el proceso de corrección de los pliegos de imprenta de la edición aldina ${ }^{25}$.

La edición giuntina sigue de manera mayoritaria este nivel de corrección al que fue sometido el texto de Giocondo. Entre la tipología de correcciones de $\mathbf{a}^{\mathbf{c}}$ que $\mathbf{g}$ secunda podemos identificar las siguientes ${ }^{26}$ :

${ }^{24}$ Errata, quae uel inter impressionem contigerunt, uel impresso uolumine deprehendimus, corrigenda sic.

25 Estas correcciones de la edición aldina, que representan un ejercicio de emendatio ope codicum et ingenii, tienen en muchos casos base en la tradición manuscrita e impresa anterior e incluso en la propia actividad conjetural del corrector o correctores. No cabe descartar que en ellas interviniera el propio Aldo, que solía revisar los textos durante el proceso de impresión de los pliegos. Cf. Al respecto A. Moreno Hernández, La edición aldina del Bellum Gallicum de César. La historia del texto en época postincunable, e. p.

${ }^{26}$ Las siglas utilizadas son las siguientes:

A) Para la tradición manuscrita del $B . G .: \omega=$ consenso de las clases a y $\mathbf{b} ; \mathbf{a}=$ consenso de los códd. AQBMSLN; $\chi=$ consenso de los códd. AQ; $\varphi=$ consenso de los códd. BMSLN; $\beta=$ consenso de los códd. TVUR; $\pi=$ consenso de los códd. TV; $\rho=$ consenso de los códd. UR.

B) Para las ediciones incunables (1469-1499): edd. $p r$. = consenso entre las ediciones incunables; $r=$ Roma 1469 (Giovanni Andrea Bussi); v = Venecia 1471 (Nicolas Jenson); $\mathbf{m}=$ Milán, 1477 (Antonius Zarotus); $\mathbf{t}=$ Treviso 1480 (Michael Manzolus); $v^{*}=$ Venecia 1482 (Octavianus Scotus); $v^{r}=$ Venecia 1490 (Theodorus de Ragazonibus); $\mathbf{v}^{\mathbf{P}}=$ Venecia 1494 (Philippus Pincius); $\mathbf{v}^{\mathbf{r}}=$ Venecia 1499 (Benedictus Fontana). 


\section{A) Correcciones gramaticales}

a) Alteraciones en los casos:

1.7.3 principum a $\mathbf{r}$ edd. pr. a principem $\pi \mathbf{b} \mathbf{a}^{\mathbf{c}} \mathbf{g} \mathbf{a}^{2}$ edd. post.

3.1.6 flumen $\alpha$ a flumine $\beta$ edd. $p r . \mathbf{a}^{c} \mathbf{g} \mathbf{a}^{2}$

6.29.3 turri $\mathbf{v}^{\mathbf{s}} \mathbf{v}^{\mathbf{f}}$ a turrim $\alpha$ rvt $\mathbf{a}^{\mathbf{c}} \mathbf{g}$ turrem $\beta$

8.11.1 locis $\alpha \rho$ edd. pr. a loci $\pi \mathbf{a}^{\mathbf{c}} \mathbf{g}$ edd. post.

8.15.1 confisi loco a confisi loci $\omega$ edd. pr. $\mathbf{a}^{\mathbf{c}} \mathbf{g}$ (edd.)

b) La voz de los infinitivos:

3.2.4 sustinere $\varphi$ edd. pr. a sustineri $\chi \beta \mathbf{a}^{\mathbf{c}} \mathbf{g} \mathbf{a}^{2}$

3.13.6 sustinere $\alpha$ a sustineri $\beta \mathbf{a}^{\mathrm{c}} \mathbf{g} \mathbf{a}^{2}$

c) Cambio de singular por plural:

1.21.1 cognosceret $\omega$ edd. pr. a cognoscerent $\mathbf{N}^{2} \mathbf{S} \mathbf{a}^{\mathbf{c}} \mathbf{g} \mathbf{a}^{2}$ edd. post.

3.3.2 possent $\omega$ edd. pr. a posset $\mathbf{a}^{\mathbf{c}} \mathbf{g} \mathbf{a}^{2}$

7.59.5 instabat $\varphi \mathrm{Vr} \mathbf{r t} \mathbf{v}^{\mathrm{s}} \mathbf{a}$ instabant $\chi \mathbf{T} \mathbf{v v}^{\mathrm{f}} \mathbf{a}^{\mathrm{c}} \mathbf{g} \mathbf{a}^{2}$

d) Correcciones de modos y tiempos verbales. Cuando a adopta una corrección de las ediciones incunables frente a la tradición manuscrita, $\mathbf{a}^{\mathbf{c}}$ recupera la lectura de ésta, y g la secunda:

2.4.8 habebantur $\beta \mathbf{a}$ habeantur $\alpha \mathbf{a}^{\mathrm{c}} \mathbf{g}$

2.29.4 reliquerant $\boldsymbol{u} \mathbf{r v} \mathbf{v}^{\mathbf{a}} \mathbf{a}$ reliquerat $\mathbf{t} \mathbf{v}^{\mathbf{s}}$ reliquerunt $\omega \mathbf{a}^{\mathrm{c}} \mathbf{g}$

2.23.1 constiterunt $\mathbf{r v t v ^ { \prime }} \mathbf{a}$ constituerunt $\mathbf{v}^{\mathbf{s}}$ constiterant $\omega \mathbf{a}^{\mathbf{c}} \mathbf{g}$

e) Formas contractas en el perfecto:

3.8.1 consuerunt $\omega$ a consueuerunt $\mathbf{a}^{c} \mathbf{g} \mathbf{a}^{2}$

f) Tratamiento de las preposiciones:

3.1.2 cum portoriis $\alpha$ edd. $p r$. a portoriis $\beta \mathbf{a}^{\mathrm{c}} \mathbf{g} \mathbf{a}^{2}$

g) Alteraciones en las conjunciones:

6.34.6-7 audacia, et $\mathbf{N}^{c}$ edd. pr. a audacia, ut $\omega \mathbf{a}^{c} \mathbf{g} \mathbf{a}^{2}$ audacia. at recc.

7.69.5 sub muroque $\beta$ QLN a sub muro quae $\mathbf{A B M S ~} \mathbf{a}^{\mathbf{c}} \mathbf{g} \mathbf{a}^{2}$

C) Para las ediciones del s. XVI: $\mathbf{a}=$ Venecia 1513 (Editio Aldina); $\mathbf{a}^{\mathbf{c}}$ correctiones in $\mathbf{a} ; \mathbf{a}^{2}=$ Venecia 1518 (Altera editio Aldina); $\mathbf{a}^{3}$ Venecia 1559 (cum correctionibus Pauli Manutii); $\mathbf{b}=$ Bolonia 1504, Philippus Beroaldus (Lion 1508; 1512); $\mathbf{i}=$ Florencia 1508 (ex officina P. Giunta); $g$ = Florencia 1514 (ex officina P. Giunta); $\mathbf{l}^{\mathbf{k}}=$ Lión 1536 (Sebastianus Gryphius); p París = 1543 (Michael Vascosanus); p $^{*}=$ París 1544 (Robertus Stephanus). 


\section{B) Alternativas léxicas}

1.28.5 in partem $\omega$ edd. pr. $\mathbf{a}$ in parem $\mathbf{a}^{\mathbf{c}} \mathbf{g} \mathbf{a}^{2}$ edd. post.

1.40.10 uiderentur $\alpha \mathbf{r} \mathbf{t} \mathbf{v}^{\mathbf{s}} \mathbf{v}^{\mathbf{f}} \mathbf{a}$ auderent $\beta \mathbf{v} \mathbf{a}^{\mathbf{c}} \mathbf{g} \mathbf{a}^{2}$ edd. post.

1.45.2 $2^{28}$ posuisset $v^{f} \mathbf{a}$ imposuisset $\omega \mathbf{r v t} \mathbf{v}^{\mathbf{s}} \mathbf{a}^{\mathbf{c}} \mathbf{g} \mathbf{a}^{2}$

2.29.3 leuiter (ya corregido en $\mathbf{a}$ ) leniret $\mathbf{a}^{\mathbf{c}} \mathbf{g} \mathbf{a}^{2}$

2.4 .5 electa $\alpha$ a $e d d$. lecta $\beta \mathbf{a}^{\mathbf{c}} \mathbf{g} \mathbf{a}^{2}$

3.8.1 eo mari $\alpha$ edd. $p r$. a eodem mari $\beta \mathbf{a}^{\mathbf{c}} \mathbf{g} \mathbf{a}^{2}$

3.15 .3 commouere $\alpha$ edd. pr. a mouere $\beta \mathbf{a}^{\mathbf{c}} \mathbf{g} \mathbf{a}^{2}$

3.27.1 ciuitates a $\mathbf{p}^{\mathbf{s}}$ nationes $\omega \mathbf{a}^{\mathbf{c}} \mathbf{g} \mathbf{a}^{2}$ edd. post.

8.15.5 conderant a consederant $\beta \mathbf{a}^{c} \mathbf{g} \mathbf{a}^{2}$ consueverant $\alpha$ consuerant $e d d$. pr.

8.29.3 confluxerant a conflixerant $\omega \mathbf{a}^{\mathbf{c}} \mathbf{g} \mathbf{a}^{2}$

\section{C) Correcciones de nombres propios}

\subsubsection{Veragos a Veragros $\omega$ edd. pr. $\mathbf{a}^{\mathrm{c}} \mathbf{g} \mathbf{a}^{2}$}

3.23.5 Sartorio a Sertorio $\omega$ edd. pr. $\mathbf{a}^{\mathfrak{c}} \mathbf{g} \mathbf{a}^{2}$

3.27.1 Cosates $\pi \mathbf{a}$ Cocosates $\alpha \rho \mathbf{a}^{\mathbf{c}} \mathbf{g} \mathbf{a}^{2}$

4.1.1 Tenchetheri a Tenchtheri $\mathbf{a}^{\mathbf{c}} \mathbf{g} \mathbf{a}^{2}$

6.9.6 Vbi qui SMN a Vbi ii $\mathbf{B}^{1} \mathbf{U}$ Vbii qui $\chi \mathbf{B}^{\mathrm{c}} \mathbf{V}$ edd. pr. $\mathbf{a}^{\mathbf{c}} \mathbf{g} \mathbf{a}^{2}$

6.25.1 Tauracorum a Rauracorum $\beta \mathbf{a}^{c} \mathbf{g} \mathbf{a}^{2}$ Tauriacorum $\alpha$

7.61.329 Rhemorum a remorum $\omega$ edd. pr. $\mathbf{a}^{\mathbf{c}} \mathbf{g} \mathbf{a}^{2}$

8.6.3 $3^{30}$ ab lucio l a $\mathbf{T}$ a $\mathbf{V}$ a L. Labieno edd.pr. a a T. Labieno i $\mathbf{a}^{\mathbf{c}} \mathbf{g} \mathbf{a}^{2}$

\section{D) Variantes gráficas y fonéticas}

Habitualmente la edición aldina mantiene la grafía clásica para ae y e; sólo en un caso a confunde las grafías y $\mathbf{a}^{\mathrm{e}}$ corrige (5.55.4 publicae priuatimque a publice priuatimque $\omega$ edd. $p r . \mathbf{a}^{\mathrm{c}} \mathbf{g} \mathbf{a}^{2}$ ).

Así mismo $\mathbf{a}^{\mathbf{c}}$ corrige en dos ocasiones el dat.-abl. plural de is (3.8.2 eis $\mathbf{a}$ iis $\mathbf{a}^{\mathbf{c}} \mathbf{g} \mathbf{a}^{\mathbf{2}}$ 6.6.3 iis $\mathbf{a}$ his $\left.\mathbf{a}^{\mathbf{c}} \mathbf{g} \mathbf{a}^{2}\right)$. En otro caso, $\mathbf{a}^{\mathbf{c}}$ registra un geminación que adoptan $\mathbf{g ~ a}^{2}(6.10 .2$ aductos $\mathbf{a}$ adductos $\mathbf{a}^{\mathbf{c}} \mathbf{g} \mathbf{a}^{\mathbf{2}}$ ).

${ }^{27}$ Esta corrección de $\mathbf{a}^{\mathbf{c}}$ demuestra el valor crítico de las conjeturas al texto de a que se encubre bajo la denominación de Errata. La propuesta de parem por partem viene a solventar de forma muy plausible la sintaxis y el sentido de la frase y es asumida de forma generalizada por los editores críticos modemos.

${ }^{2 k}$ En este caso el error que comete la edición veneciana de 1499 y que reproduce a se explica por una haplografía propiciada por el contexto paleográfico (stipendium imposuisset). El resto de las ediciones incunables. $\mathbf{a}^{\mathbf{c}}$ y g, corrigen de acuerdo con la tradición masnucrita mayoritaria.

${ }_{24}$ a corrige la lectio recepta para introducir el nombre de los $R$ hemi, carente de sentido en este contexto. $\mathbf{a}^{\mathbf{c}}$ corrige según la tradición mayoritaria, y g la asume.

${ }^{30}$ a sigue aquí la lectura común de las ediciones incunables, pero a corrige a la vista de la edición de Giunta de Florencia 1508. Esta es la lectura que enmienda la corrupción manuscrita y es adoptada por $\mathrm{g}$ y modernamente por los editores críticos. 


\section{E) Seclusiones y adiciones}

a) Seclusiones

1.42.6 ei legioni a om. $\mathbf{B}^{\mathbf{c}} \boldsymbol{\beta} \mathbf{a}^{\mathbf{c}} \mathbf{g} \mathbf{a}^{2}$ ei $\alpha$

2.16.1 captiuis eorum $\beta$ a captiuis $\alpha$ edd. $p r$. $\mathbf{a}^{\mathbf{c}} \mathbf{g} \mathbf{a}^{2}$

2.28.1 et maiores natu a maiores natu $\omega$ edd. $p r . \mathbf{a}^{\mathbf{c}} \mathbf{a}^{\mathrm{c}} \mathbf{g} \mathbf{a}^{2}$

3.1.2 cum portoriis a edd. pr. a portoriis $\mathbf{b} \mathbf{a}^{c} \mathbf{g} \mathbf{a}^{2}$

3.9.6 quos neque ullam facultatem a neque ullam facultatem $\omega \mathbf{a}^{\mathbf{c}} \mathbf{g ~ a}^{2}$

3.13.6 hae siue propter lini a siue propter lini $\omega \mathbf{a}^{\mathbf{c}} \mathbf{g} \mathbf{a}^{2}$

3.13.6 posse arbitrabantur $\alpha \mathbf{a}$ arbitrabantur $\beta \mathbf{a}^{\mathbf{c}} \mathbf{g} \mathbf{a}^{2}$

3.14.4 tantum tamen a tamen $\omega \mathbf{a}^{\mathbf{c}} \mathbf{g} \mathbf{a}^{2}$

7.68.3 si qua maxima a qua maxima $\alpha \mathbf{a}^{\mathbf{c}} \mathbf{g} \mathbf{a}^{2}$

b) Adiciones

3.9.1 quod ipse aberat $\omega$ edd. $p r$ ille aberat a quod ille aberat $\mathbf{a}^{\mathbf{c}} \mathbf{g} \mathbf{a}^{2}$ edd. post.

3.9.6 insulas nouisse $\mathbf{a}$ insulasque nouisse $\mathbf{a}^{c} \mathbf{g ~ a}^{2}$

3.10.1 erant difficultates $\mathbf{a}$ erant hae difficultates $\omega \mathbf{a}^{c} \mathbf{g} \mathbf{a}^{2}$

3.13.3 naues totae a nauesque totae $\omega \mathbf{a}^{\mathbf{c}} \mathbf{g} \mathbf{a}^{2}$

\section{G) Cambios en la puntuación}

a) Alteración en la colocación de comas:

3.1.5 in ualle non magna, adiecta planicie, $\mathbf{a}$ in ualle, non magna adiecta planicie, $\mathbf{a}^{\mathbf{c}} \mathbf{g} \mathbf{~ a}^{\mathbf{2}}$

3.9.4 concisa, aestuariis nauigationem a concisa aestuariis, nauigationem $\omega \mathbf{v} \mathbf{a}^{c} \mathbf{g ~ a}^{2}$ aestuariam nauigationem $\mathbf{r t} \mathbf{v}^{\mathbf{s}} \mathbf{v}^{\mathbf{t}}$

8.32.2 fuerat, eius natura a fuerat eius, natura $\mathbf{a}^{c} \mathbf{g} \mathbf{a}^{2}$

b) Supresión de comas de a:

7.48.4 simul, et curso a simul et cursu edd. pr. $\mathbf{a}^{\mathbf{c}} \mathbf{g} \mathbf{a}^{2}$

$8.14,2$ oppressi, luce a oppressi luce $\mathbf{a}^{\mathbf{c}} \mathbf{g} \mathbf{a}^{2}$

8.16.3 progressi, hostes a progressi hostes $\mathbf{a}^{\mathrm{c}} \mathbf{g} \mathbf{a}^{2}$

8.46.6 sustinuerat, fidelitate a sustinuerat fidelitate $\mathbf{a}^{\mathbf{c}} \mathbf{g} \mathbf{a}^{2}$

c) Inserción de comas ausentes en a:

7.80.1 nouerit equitatum $\mathbf{t} \mathbf{v}^{\mathbf{s}} \mathbf{v}^{\mathfrak{q}} \mathbf{a}$ nouerit, equitatum $\mathbf{r v} \mathbf{a}^{\mathbf{c}} \mathbf{g} \mathbf{a}^{2}$

8.31.2 tempore eodem a tempore, eodem $\mathbf{a}^{\mathbf{c}} \mathbf{g} \mathbf{a}^{2}$

8.31.4 Carnutum aduentu a Carnutum, aduentu $\mathbf{a}^{\mathbf{c}} \mathbf{g} \mathbf{a}^{2}$

8.41.2 periculo e regione a periculo, e regione $\mathbf{a}^{c} \mathbf{g ~ a}^{2}$

d) Sustitución de un punto de a por una coma:

3.22.1 appellant. Quorum $\mathbf{r v t} \mathbf{v}^{\mathbf{s}} \mathbf{a}$ appellant, quorum $\mathbf{v}^{\mathbf{f}} \mathbf{a}^{\mathbf{c}} \mathbf{g} \mathbf{a}^{2}$ 


\section{H) Erratas tipográficas de a}

\subsection{7 exercitassimi a exercitatissimi $\mathbf{a}^{\mathbf{c}} \mathbf{g} \mathbf{a}^{2}$}

3.6.2 superiobus $\mathbf{a}$ superioribus $\mathbf{a}^{c} \mathbf{g} \mathbf{a}^{2}$

6.10.2 aductos $\mathbf{a}$ adductos $\mathbf{a}^{c} \mathbf{g} \mathbf{a}^{2}$

6.31.1 dubium clam dimissis a dubium est. Sed certe clam dimissis ${ }^{31} \mathbf{a}^{\mathbf{c}} \mathbf{g} \mathbf{a}^{2}$

8.8.3 impedimentorm a impedimentorum $\mathbf{a}^{\mathbf{c}} \mathbf{g} \mathbf{a}^{2}$

8.18.4 proter a propter $\mathbf{a}^{\mathbf{c}} \mathbf{g} \mathbf{a}^{2}$

Estas lecturas demuestran, por consiguiente, que la edición giuntina parece haber asumido escrupulosamente las correcciones propuestas en forma de Errata al texto de Giocondo, muchas de las cuales responden a un criterio filológico bien fundado, ya sean enmiendas ope codicum (cf. 1.40.10 uiderentur $\alpha \mathbf{r} \mathbf{t} \mathbf{v}^{\mathbf{s}} \mathbf{v}^{f} \mathbf{a}$ auderent $\beta \mathbf{v} \mathbf{a}^{\mathbf{c}} \mathbf{g} \mathbf{a}^{2}$ edd. post.), ya sean ope ingenii (1.28.5 in partem $\omega$ edd. pr. $\mathbf{a}$ in parem $\mathbf{a}^{\mathrm{c}} \mathbf{g} \mathbf{a}^{2}$ edd. post. ${ }^{32}$.

\section{DivergenCiAS DE g RESPECTO A LA EDICIÓN ALDINA}

Pero por debajo de este mimetismo entre ambas ediciones puede descubrirse la labor crítica que desarrolla g, interviniendo sobre el texto aldino en distintos niveles. Según la índole de estas divergencias podemos distinguir diversas formas de intervención:

\subsection{Variantes críticas con base en la tradición manuscrita o impresa}

La lectura que hace $\mathbf{g}$ de a no sólo le permite corregir errores separativos de esta edición ${ }^{33}$, sino reconsiderar un buen número de lecturas aldinas, reconsideración que la edición giuntina lleva a cabo siempre en la misma dirección: recuperando lecturas de la tradición manuscrita e impresa anterior. Se trata, por tanto, de una labor de emendatio ope codicum et editionum, que se advierte en los siguientes niveles:

\section{A) Revisión de lecturas de la edición aldina}

a) Rechazo de lecturas de a con base en la tradición manuscrita e impresa anterior:

6.16.5 innocentium $\alpha \pi$ edd. $p r$. i a innocentiam $\rho \mathbf{g} \mathbf{a}^{2}$

31 En este caso, el salto de página ha condicionado el error: a salta de dubium a clam dimissis; $\mathbf{a}^{\mathrm{c}}$ marca la omisión de est sed certe, que sin embargo se recoge en el reclamo del fol. $72 \mathrm{v}$ de a.

${ }^{32}$ Esta corrección de $\mathbf{a}^{\mathrm{c}}$-asumida por $\mathrm{g}$ - es una hallazgo crítico incuestionable, que viene a resolver la construcción y el sentido del pasaje y revela la envergadura de la labor crítica que se esconde bajo estas aparentes errata a la edición aldina. No en vano parem es asumida unánimemente por los editores modemos.

336.10 .4 peste aquam a posteaquam $\omega \mathbf{g} \mathbf{a}^{2} ; 7.15 .3$ denfedi a defendi $\mathbf{g} \mathbf{a}^{2}$.

Así mismo, a pesar de la calidad de g, no está exento su texto de algunos errores separativos:

7.1.4 Aruernus $\omega \mathbf{a} \mathbf{a}^{2}$ Aruernis $\mathbf{g}$

7.55.4 magistratum $\omega \mathbf{a ~ a ~}^{2}$ magistrum $\mathrm{g}$

3.10.2 sibi idem licere $\omega$ a idem sibi licere $\mathbf{g}$ 
g sigue una lectura minoritaria, representada por los mss. de la familia $\rho$, fruto de la deformación de la tradición de $\alpha \pi$, críticamente preferible («descienden a los suplicios de los inocentes") y que es adoptada por las ediciones incunables y la propia edició aldina.

\subsection{9 neu suis $\operatorname{sint} \alpha \mathbf{i}$ a $\mathbf{a}^{2}$ ne suis $\operatorname{sint} \beta \mathbf{g}$}

La edición aldina ha reproducido en este caso una lectura de parte de la tradición manuscrita e impresa que revela una clara anticipatio respecto a neu Romanis proposi$t a \ldots$ que sigue a este pasaje. La edición de Giunta de 1514 ha optado por decantarse por la variante $n e$, avalada por la tradición de $\beta$ y portadora de la lectura más viable críticamente.

\subsection{3 magnis $\mathrm{Lr}$ edd. $p r$ a magnisque $\omega$ i $\mathrm{g} \mathrm{a}^{2}$}

g vuelve a apartarse de la lectura que había introducido a a partir de una parte de la tradición, asumiendo la lección común de los manuscritos que establece la concordancia entre spe libertatis y magnis praemiis, sin problemas críticos. a había adoptado una corrección presente en algunos recentiores y en las ediciones incunables, que críticamente no resulta pertinente.

b) Rechazo de correcciones de la edición aldina sin base en la tradición manuscrita anterior:

En general se trata de variantes procedentes de la tradición manuscrita mayoritaria frente a correcciones de $\mathbf{a}$. En efecto, $\mathbf{g}$ revisa algunas correcciones del texto de Giocondo para recuperar la lectura más extendida en la tradición manuscrita e impresa anterior:

\subsection{3 proximi a proximique $\omega \mathbf{b}$ i $\mathbf{g} \mathbf{a}^{2} e d d$. pr., edd. post.}

La seclusión de la enclítica por parte de a permite establecer un paralelismo con fortissimi sunt Belgae y a la vez una uariatio respecto al más cercano minimeque ${ }^{34}$. Sin embargo la edición de Giunta rechaza esta corrección y recupera la lección de la tradición manuscrita e impresa anterior. Las ediciones aldinas posteriores, así como las restantes ediciones del XVI, desechan la lectura de a y editan proximique.

\subsection{2 illum $\omega$ edd.pr. $\mathbf{g} \mathbf{a}^{2} \mathbf{a}^{3}$ Caesarem i a p $\mathbf{p}^{\mathrm{v}}$}

g, seguida por $\mathbf{a}^{2} \mathbf{a}^{3}$, desecha la corrección de a, que tiene por objeto la explicitación del complemento para evitar cualquier ambigüedad en la identificación del pronombre.

1.31.1 secreto et in oculto $\mathbf{a} \mathbf{p}^{\mathrm{v}} \mathbf{p}^{\mathrm{s}}$ secreto in oculto $\omega \mathbf{g} \mathbf{a}^{2} \mathbf{a}^{3}$ secreto $\mathbf{r} \mathbf{v}$ secreto et oculto t $v^{s} v^{f} \mathbf{i}$

34 La lectura proximi estaría ya en el Carrariensis, según la anotación de Bruto en su libellus. editado en la edición de los Commentarii de Lyón 1560. 
La edición de 1514 , a la que siguen las ediciones aldinas $\mathbf{a}^{2}$ y $\mathbf{a}^{3}$, recupera la lectio recepta frente a la corrección a, que se inscribe dentro del proceso de corrección que atestiguan las ediciones incunables ${ }^{35}$. El proceso de gestación de la enmienda aldina se explica teniendo en cuenta la corrección que algunas ediciones incunables de la década de los 80 introducen: a ha sincretizado elementos de la lectio recepta de la tradición impresa (in oculto), omitida por rv, con la enmienda presente en $\mathbf{t} \mathbf{v}^{\mathbf{s}} \mathbf{v}^{\mathbf{f}}$ (et), fusionándolas en su edición (secreto et in oculto).

\subsection{7 in intercluso mari a in concluso mari $\omega$ edd. pr., b i g edd.post.}

g sigue la lectio recepta, que se aparta de una corrección de a que no encuentra ningún respaldo en la tradición impresa e incunable (cf. los pasajes paralelos de B. Alexandrinum $43.1^{36}$ y Livio $44.14 \cdot 10^{37}$ ).

3.9.7 atque in uastissimo ac apertissimo oceano a atque in uastissimo atque apertissimo oceano $\varphi \mathbf{g}$

g revisa la corrección de a siguiendo una parte de la tradición manuscrita que tiene su base en los mss. de la familia $\varphi$, mientras que el segundo atque está omitido en la clase $\beta$.

\subsection{3 qua ratione b i a quam rationem $\omega \mathbf{g} \mathbf{a}^{2}$}

La edición giuntina se aparta de la corrección de a, poco plausible tanto desde el punto de vista gramatical como del sentido, para recuperar la lectura mayoritaria de la tradición.

\subsection{2 hi $\beta$ et $\alpha$ edd. pr., edd. post. ii a}

a se aparta de las ediciones incunables para revisar la lectura de $\beta$, deformada en $i i$ in embargo $\mathrm{g}$ recupera la variante de $\alpha$ y de las ediciones incunables.

\subsection{3 erat pulsus $e d d . p r$. i a erant pulsi $\omega \mathbf{g} \mathbf{a}^{2}$}

El texto de Giocondo, que sigue la lectura exendida en las ediciones incunables presenta el verbo en singular, como si el sujeto fuera Caesar, y atraido por el próximo adhortatus...instituit. Sin embargo el sujeto no expreso de esta oración introducida por quod es claramente hostes, de manera que $\mathrm{g}$ retoma la tradición manuscrita y lee correctamente el verbo en plural.

35. Según Meusel (Tabula coniecturarum, incluida dentro de su Lexicum Caesarianum, Berlín 1887-1893) las primeras ediciones omiten in oculto, pero en realidad esto sólo ocurren en la edición príncipe de Roma 1469 y en la siguiente, de Venecia 1471, mientras que la serie de ediciones incunables posteriores a Treviso 1480 registran la conjetura secreto $\langle$ et $>$ oculto.

${ }^{36}$ Gabinius ut in Illyricum uenit hiberno tempore anni ac difficili siue copiosiorem prouinciam existimans siue multum fortunae uictoris Caesaris tribuens siue uirtute et scientia sua confisus qua saepe in bellis periclitatus magnas res et secundas ductu auspicio que suo gesserat neque prouinciae facultatibus subleuabatur quae partim erat exinanita partim infidelis neque nauibus intercluso mari tempestatibus commeatus supportare poterat magnis que difficultatibus coactus non ut uolebat sed ut necesse erat bellum gerebat.

${ }^{37}$ tertium se annum multa eius incommoda belli sentire mari intercluso; + inopiam insulam inopem miss - - <ma > ritimis iuuetur colendi itaque commeatibus + 


\section{B) Rechazo de correcciones de $\mathbf{a}^{\mathrm{c}}$}

La tendencia de la edición de 1514 es reproducir las lecturas de a y seguir las correcciones de $\mathbf{a}^{\mathbf{c}}$, pero en algunos casos $\mathbf{g}$ se aparta de ambas para recuperar una variante de la tradición manscrita y de las primeras ediciones:

\subsection{2 graueretur a grauarentur $\mathbf{a}^{\mathrm{c}}$ grauaretur $\omega$ edd. pr. i $\mathbf{g} \mathbf{a}^{2} e d d$. post.}

El subjuntivo en singular de la lectio recepta se ajusta sin lugar a dudas a la construcción introducida por $u t$ y coordinada con el siguiente neque putaret. El plural de $\mathbf{a}^{\mathbf{c}}$ presupone un sujeto distinto al implícito (Ariovisto).

1.42.6 et nunc ad equum rescribere $e d d . p r$. $b$ i a et ad equum rescribere $\mathbf{a}^{c}$ ad equum rescribere $\omega \mathbf{g} \mathbf{a}^{2} e d d$. post.

a sigue la tradición de las ediciones incunable y de Beroaldo, pero la corrección de $\mathbf{a}^{\mathbf{c}}$ recupera la lectio recepta de los mss., que continúan las ediciones incunables. La corrección de la edición aldina viene a desechar una conjetura de los recentiores para volver a la tradición manuscrita. Sin embargo, g retoma la tradición manuscrita mayoritaria.

\subsection{2 consuerant $\mathbf{L} \mathbf{p} \mathbf{r}$ a consueuerunt $\mathbf{a}^{c}$ consueuerant $\alpha \mathbf{r} \mathbf{v t v}^{\mathbf{s}} \mathbf{v}^{\mathrm{f}} \mathbf{g} \mathbf{a}^{\mathrm{c}}$ edd. post.}

$\mathbf{g}$ recupera la lectura de la tradición manuscrita frente a $\mathbf{a}^{\mathbf{c}}$, que propone un perfecto frente a toda la tradición, en un contexto narrativo en pasado fuit... uolebat, que no exige alterar el tiempo verbal. De hecho la edición de Giunta recupera la lectio recepta y a partir de ahí se generaliza en las ediciones incunables.

5.52.1 uidebat. Omnibus suis incolumis copiis, eodem die edd. pr. uidebat, omnibus suis incolumibus copiis. Eodem die a uidebat, omnibus suis incolumibus copiis, in eodem die $\mathbf{a}^{\mathbf{e}}$ uidebat, omnibus suis incolumibus copiis, eodem die $\mathbf{g ~ a}^{2}$ edd. post. uidebat-, omnibus suis incolumibus copiis, eodem die (edd.)

Pasaje sospechoso sometido a una constante revisión de la puntuación ${ }^{38}$. Las ediciones incunables colocan punto tras uidebat y una coma tras copiis; a ha alterado esta puntuación, con coma tras uidebat y punto tras copiis, punto que $\mathbf{a}^{\mathbf{c}}$ elimina, recuperando la coma, e introduce la preposición in para la construcción en ablativo, propuesta sin precedentes en la tradición impresa ni manuscrita. Sin embargo la tradición aldina posterior no sigue esta propuesta, adoptando la variante de la edición de Giunta de 1514, proxima a la tradición representada por la clase $\alpha$.

38 Las dos clases leen en este pasaje:

uidebat omnibus suis incolumibus copiis eodem die $\alpha$ uidebat omnibus suis incolumibus eodem die $\beta$ 


\subsection{Otros niveles de corrección al texto de la edición aldina}

La revisión que lleva a cabo $\mathrm{g}$ del texto veneciano de 1513 no se limita a la recuperación de lecturas con base en la tradición anterior, sino que se verifica a otros muchos niveles, algunos de ellos muy reveladores de la voluntad del impresor florentino de depurar el texto fijado por Giocondo en la edición aldina. Podemos apuntar entre ellos los siguientes:

A) Discrepancias de carácter grafico-fonético: A pesar de que g adopta de manera abrumadora las variantes gráficas de $\mathbf{a}$, hemos podido detectar algunos casos de divergencia en este nivel, que afectan a algunos fenómenos habituales de vacilación en la notación latina ${ }^{39}$.

B) Notación de las mayúsculas: La edición aldina muestra indicios claros de un uso regular de la mayúscula en determinados contextos. La edición de Giunta sigue a su modelo mayoritariamente, si bien se advierte un grado notable de intervención con el propósito de codificar con más precisión su utilización en determinadas posiciones.

Así se constata la tendencia de $\mathbf{g}$ de generalizar la mayúscula tras punto, contexto donde a se muestra todavía extremadamente vacilante (7.24.1 potest. his tot a $\mathbf{a}^{2}$ potest. His tot $\mathbf{g}$ ), o bien a regularizar su uso en gentilicios ${ }^{40}$ y nombres de lugar ${ }^{41}$, así como en la codificación de la mayúscula respecto a términos relacionados con el poder y sus instituciones y con cargos civiles y militares ${ }^{42}$, o bien con otros vocablos ${ }^{43}$.

C) Modificaciones en la puntuación. La edición de Giunta profundiza en la preocupación mostrada en la edición de Giocondo por revisar la puntuación del texto cesariano, sobre todo en la colocación de las comas. Hemos localizado algunos ejemplos en los que el texto de 1514 corrige a la edición aldina en varios pasajes en los que la modificación de la coma resulta congruente con la construcción y con el sentido, y es asumida por los editores modernos:

\subsection{6 restitutis nouis a restitutis, nouis $\mathbf{g} \mathbf{a}^{2}$ edd.}

${ }^{39}$ La vacilación $-t i$ - $c i$ ante vocal $\left(6.3 .6\right.$ pronuntiata a $\mathbf{a}^{2}$ pronunciata $\mathbf{g} ; 7.20 .8$ pronuntiare a $\mathbf{a}^{2}$ pronunciare g; 6.10.1 denuntiare $\mathbf{a} \mathbf{a}^{2}$ denunciare $\mathrm{g} ; 8.15 .5$ pronuntiato a $\mathbf{a}^{2}$ pronunciato $\mathrm{g}$ ), la notación de ae/e (6.11.4 egeret $\mathbf{a} \mathbf{a}^{2}$ aegeret $\mathbf{g} ; 6.24 .4$ egestate $\mathbf{a} \mathbf{a}^{2}$ aegestate $\mathbf{g} ; 7.14 .10$ aestimari $\mathbf{a} \mathbf{a}^{2}$ estimari $\mathbf{g} ; 8.29 .3$ cedentibus $\mathbf{a} \mathbf{a}^{2}$ caedentibus $\mathbf{g})$ o bien $\left(6.17 .3\right.$ ceperunt $\mathbf{T} \mathbf{r} \mathbf{a} \mathbf{a}^{2}$ coepenunt $\mathbf{g} ; 8.30 .1$ cepisse $\mathbf{a} \mathbf{a}^{2}$ coepisse $\left.\mathbf{g}\right), o$ i/y (7.11.8 desyderatis $\mathbf{a} \mathbf{a}^{2}$ desideratis $\left.\mathbf{g}\right), m / n$ ante consonante $\left(8.13 .1\right.$ utrorunque $\mathbf{a} \mathbf{a}^{2}$ utrorumque $\mathbf{g} ; 8.39 .2$ contemnebat $\mathbf{a} \mathbf{a}^{2}$ comtemnebat $\mathbf{g}$ ); la notación de $h$ (8.43.5 exaustus a $\mathbf{a}^{2}$ exhaustus $\mathbf{g}$ ).

${ }^{40}$ 6.35.4 usipetes a $\mathbf{a}^{2}$ Vsipetes g; 7.7.4 uolcis, artomicis a $\mathbf{a}^{2}$ Volcis, Artomicis $\mathbf{g} ; 7.9 .4$ lingones $\mathbf{a} \mathbf{a}^{2}$ Lingones g; 8.23.6 romani a $\mathbf{a}^{2}$ Romani $\mathbf{g} ; 3.14 .7$ gallicis a Gallicis $\mathbf{g} ; 7.14 .1$ genabi a Genabi $\mathbf{g} \mathbf{a}^{2}$. En cambio en 7.59.1 Ligere cba $\mathbf{a}^{2}$ es banalizado en ligere $\mathrm{g}$ (cf. Ligeri $\mathbf{f}$ ).

417.8 .2 gebenna a Gebenna $\mathrm{g} \mathbf{a}^{2}$; pero en cambio 7.8.3 Gebenna a $\mathrm{g}$; $\mathbf{a} 6.31 .3$ (cf. 8.31.4) oceano a $\mathrm{a}^{2}$ Oceano $\mathbf{g}$-pero en 6.33 .1 Oceanum $\mathbf{a} \mathbf{g}-$ ).

42 Imperium se nota ocasionalmente con mayúscula en a, pero g lo escribe con minúscula (7.4.6.9 Imperium $\mathbf{a} \mathbf{a}^{2}$ imperium $\mathbf{g}$ ), al igual que imperator (7.1.7 (cf. 7.52.3) Imperator a $\mathbf{a}^{2}$ imperator $\mathrm{g}$, pero no siempre, cf. 8.19.4 Imperatorem a $\mathbf{g}$ ); en cambio senatum, sobre la cual vacila $\mathbf{a}$, se escribe preferentemente con mayúscula en $\mathbf{g}$ (7.33.2 Senatum a $\mathbf{g}$, pero 7.33.3 senatu a Senatu $\mathbf{g} \mathbf{a}^{2}$ ); algunos cargos que a escribe con mayúscula se notan en miníscula en $\mathrm{g}$ (8.48.3 Praefectum a $\mathrm{a}^{2}$ praefectum g; 8.55.1 Duci a duci $\mathrm{g}$.

${ }^{43}$ otras discrepancias pueden verse en 6.18.1 ab Dite Patre $\mathbf{a} \mathbf{a}^{2}$ ab Dite patre $\mathbf{g} ; 7.19 .3$ marte $\mathbf{a} \mathbf{a}^{2} \mathbf{~ M a r t e ~} \mathbf{g}$; 7.26.4 Timor $\mathbf{a} \mathbf{a}^{2}$ timor $\mathbf{g} ; 7.41 .5$ solis $\mathbf{a} \mathbf{a}^{2}$ Solis $\mathbf{g} ; 8.55 .2$ Bello a bello $\mathbf{g}$. Algunas menciones que a marca con mayúscula, son respetadas por $\mathbf{g}$ (7.73.9 Stimulos nominabant $\mathbf{a} \mathbf{g} \mathbf{a}^{2}$ ) 
7.72.2 aut noctu ad munitiones hostium... aduolaret $\mathbf{a} \mathbf{a}^{2}$ aut noctu ad munitiones, hostium aduolaret $\mathbf{g}$

8.24.4 si quos fortuna fecisset reliquos nullum reditum...haberet $\mathbf{a} \mathbf{a}^{2}$ si quos fortuna fecisset reliquos, nullum reditum...haberet $\mathbf{g}$

8.31.2 eodem instante, Dumnaco possent $\mathbf{a} \mathbf{a}^{2}$ eodem instante Dumnaco possent $\mathbf{g}$

D) Nombres propios: $\mathbf{g}$ sigue las lecturas de $\mathbf{a}$, incluso sus banalizaciones (7.55.4 [cf. 7.67.4] Conuictolitanem ag Conuictolitauem $\beta \mathbf{N}$ Conuictolitabim c BMSL), si bien en alguna ocasión la edición giunta introduce una corrección sistemática en algún nombre propio: 6.5.1 Treuerorum $\alpha \mathbf{a} \mathbf{a}^{2}$ Treuirorum $\beta \mathbf{g}$ (cf. 6. 5.4.6; 6.3; 7.1; 8.7; 9.1.6; $29.4 ; 32.1 ; 8.25 .1 ; 8.45 .1 ; 52.1)$, y sólo excepcionalmente sigue a a (6.9.1.5. Treueris a g) ${ }^{44}$.

E) Abreviaturas. Ocasionalmente $\mathbf{g}$ recurre al uso de formas de abreviaturas diferentes a a: 7.1 .3 populi Romani a $\mathbf{a}^{2}$ populi Ro. G; 6.1 .4 reipub. a $\mathbf{a}^{2}$ Reip. G. Tambien en los ordinales: 6.64.1 quindecim millia $\mathbf{a} \mathbf{a}^{2} \mathrm{XV}$ millia $\mathrm{g}$.

\section{Conclusiones}

Del análisis de la edición del Bellum Gallicum de Philippo de Giunta de 1514 (g) y su contraste con la edición aldina del año anterior (a) se desprende que, desde el punto de vista del texto que trasmite, $\mathbf{g}$ ha sido elaborada con un cuidado extremo y se ha basado sin lugar a dudas en la edición aldina, cuyo texto - tanto el preparado por Giocondo (a) como las correcciones que preceden éste $\left(\mathbf{a}^{\mathbf{c}}\right)$ - asume casi integralmente en todos sus niveles, pues tiende a reproducir las lecturas de la edición aldina, sus tendencias gráficas y pautas de puntuación, a partir de un formato de composición similar - utilizado en la edición de Giunta de 1508 - en $8^{\circ}$ con caracteres cursivos y caja de 30 líneas por página, sin títulos marginales y con disposición continua del texto, aunque para evitar la impresión de que se trata de una mera reproducción de su modelo procura evitar en la composición la coincidencia de fin de línea y plana.

No obstante, bajo este carácter mimético, $\mathbf{g}$ interviene en el texto establecido por la edición aldina mediante el ejercicio de una incipiente labor crítica, revisando un buen número de lecturas de a, revisión que desde el punto de visto crítico tiene una orientación predominante: la adopción de lecturas mayoritarias en la tradición manuscrita e impresa anterior frente a variantes y correcciones de a y $\mathbf{a}$. La edición giuntina no es una mera reproducción de la aldina, sino que lleva a cabo un ejercicio de emendatio ope codicum et editionum, desechando propuestas de a para recuperar la lectio recepta de la tradición. No hay, sin embargo, trazas de una crítica conjetural propia del editor florentino, es decir, de una emendatio ope ingenii.

La importancia de este proceso para la historia del texto de los Commentarii en las primeras décadas del Quinientos estriba en que el texto resultante de $\mathbf{g}$ va a ejercer un in-

44 7.63.7 Treuiri 2. $e d d$. pr. a Treueri $\omega \mathbf{a}^{\mathrm{c}} \mathbf{g} \mathbf{a}^{2}$. En este caso $\mathbf{a}^{\mathrm{c}}$ corrige siguiendo la lectura anterior (Treueri $1 . "$ ) del mismo pasaje. 
flujo muy importante sobre la segunda edición aldina de $1519^{45}$ y a partir de ella, en las principales ediciones postincunables hasta 1559, cuando se publica la edición de Paolo Manuzio, que todavía hereda el texto de Giocondo (Venecia 1559, identificada como $\mathbf{a}^{3}$ ).

APÉNDICE: Descripción de la edición

Titulo: Commentaria Caesaris, prius a Jocundo impressioni data, posterius a nobis diligentissime revisa... In his autem commentariis continentur: (Caesaris) de bello Gallico libri VIII, de bello civili Pompeiano libri IV. - (A. Hirtii) de bello Alexandrino liber I, de bello Africano liber I, de bello Hispaniensi liber I. Nomina locorum urbiumque et populorum Galliae... latine et... gallice... Pictura totius Galliae... (cum figuris J. Jucundi.) Item est addita Hispaniae descriptio.

Colofón (285v): Florentiae, ex officina Philippi de Giunta Florentini, Anno M.D.XIIII. Men. August. Leone X. Pont. Max.

Descripción física: 12 ff. Sin numerar (dos cuadernillos a3-b8), 285 ff. numerados en el recto (i.e. 283) (error en la la foliciación: están omitidos los ff. 193 y 218) (Registro a8z8; 12 A-N8). En 8.० (16 cms.).

Caja tipográfica: la edición presenta de forma regular 30 líneas por página.

Descripciones: Adams $^{46} \mathrm{C} 27$; Decia/Giunta ${ }^{47}$ I, 52; ICCU $^{48} \mathrm{C} 127$; Index Aur. ${ }^{49}$ 128.658; CNCE (Censimento Nazionale Cinquecentine) 8148

\section{Contenido:}

A) Folios sin numeración iniciales: 6 grabados y sus correspondientes comentarios: la construcción del puente sobre el Rin (4.17); la fortificación de Avarico, capital de los bitúriges (7.22-23); la fortificación que preparó César para el cerco de Alesia (7.62-73); la forticación de Uxeloduno (8.40); y la descripción de Massilia (B.C. 2.9). Descripción de Hispania (ausente en la edición aldina) - Índice de nombres propios en latín y en francés.

\footnotetext{
45. Aunque esta segunda edición aldina $\left(\mathbf{a}^{2}\right)$ introduce algunas correcciones, en general de poca entidad: 3.28.1 pacata, Morini, a g pacate Morini $\mathbf{a}^{2}$. La mayoría de las discrepancias de $\mathbf{a}^{2}$ frente a y $\mathbf{g}$ se deben a errores en el proceso de composición y no al uso de otra fuente:

1.50.1 maioribus $\omega$ edd. pr. a g maiorbus $\mathbf{a}^{2}$

2.12.5 aggere $\omega$ edd. $p r$. a g agere $\mathbf{a}^{2}$

7.5.7 Aruernis $\omega$ edd. pr. a g Auernis $\mathbf{a}^{2}$

7.7.2 ab utrisque $\omega$ edd. pr. a $\mathbf{g}$ ab utriusque $\mathbf{a}^{2}$

7.7.4 prouincialibus $\omega$ edd. $p r$. a $\mathbf{g}$ prouicialibus $\mathbf{a}^{2}$

th Catalogue of Books printed on the Continent of Europe, 1501-1600 in Cambridge Libraries, compiled by H. M. Adams, 2 v., Cambridge 1967.

${ }^{47}$ D. Decia, / Giunti tipografi editori di Firenze, op.cit.

${ }^{4 *}$ Istituto Centrale per il Catalogo Unico delle Biblioteche Italiane e per le Informazioni Bibliografiche, $L e$ edizioni italiane del XVI secolo. Censimento nazionale, Roma, 1989.

${ }^{49}$ Index Aureliensis, Catalogus librorum sedecimo saeculo impressorum. Aureliae Aquensis, 1965.
} 
B) Corpus Caesarianum: ff. 1-285: 1r- 111r: De bello Gallico (Libri VIII); 111v187r: De bello ciuili. (Libri III); 187v-211v: De bello Alexandrino (Liber I); 212r-241r: De bello Africano (Liber I); 241v-254v: De bello hispaniensi (Liber I).

C) Índice de Raimundo Mariliano 255r-285v.

Ejemplares consultados: París, BNF J-13272, y Arsenal, magasin 8- H- 2836; British Library, Humanities C.19.f. 12.

Sobre los ejemplares conservados en las bibliotecas italianas, resulta de gran utilidad el Censimento delle edizioni italiane del XVI secolo, proyecto desarrollado por el Istituto Centrale per il Catalogo Unico delle biblioteche italiane e per le informazioni bibliografiche (ICCU), http://edit16.iccu.sbn.it/web_iccu/ihome.htm. Hasta el momento se han publicado 5 volúmenes: La edizioni italiane del XVI secolo: censimento nazionale, que desde 1985 se publica en Roma (V vol., 2005). 